\title{
Computationally efficient boundary element methods for high-frequency Helmholtz problems in unbounded domains
}

Timo Betcke, Elwin van 't Wout and Pierre Gélat

\begin{abstract}
This chapter presents the application of the boundary element method to high-frequency Helmholtz problems in unbounded domains. Based on a standard combined integral equation approach for sound-hard scattering problems we discuss the discretization, preconditioning and fast evaluation of the involved operators. As engineering problem, the propagation of high-intensity focused ultrasound fields into the human rib cage will be considered. Throughout this chapter we present code snippets using the open-source Python boundary element software BEM++ to demonstrate the implementation.
\end{abstract}

\section{Introduction}

The boundary element method (BEM) is an efficient and competitive tool to solve large-scale high-frequency Helmholtz problems in bounded or unbounded domains. Recent developments in fast matrix compression and preconditioning for boundary integral operators have pushed the computational limit of high-frequency boundary element computations such that problems in three dimensions with over a hundred wavelengths across the domain can be solved on a single workstation [48]. Furthermore, the availability of high-level software libraries allows for a convenient implementation of different boundary integral formulations [42]. This combination

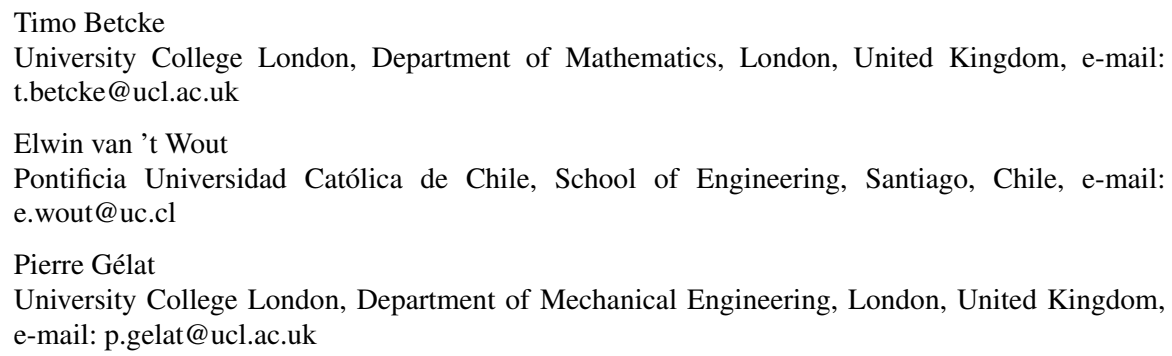


makes it possible to solve large-scale problems of engineering interest effectively with the BEM.

This chapter will deal with exterior scattering of sound waves. In this case, a bounded domain $\Omega^{-} \subset \mathbb{R}^{3}$ is immersed in a homogeneous unbounded region $\Omega^{+}:=$ $\mathbb{R}^{3} \backslash \overline{\Omega^{-}}$and excited by a harmonic wave with a fixed wavenumber $k$. Notice that the object has to be bounded but not necessarily connected. The main objective is the computation of the total wave field $u^{\text {tot }}$ obtained from the scattering of an incident wave field $u^{\text {inc }}$ at the object. For rigid objects, we have a sound-hard condition at the boundary $\Gamma$, which is assumed to be Lipschitz continuous with unit normal direction $\hat{\mathbf{n}}$ outward pointing. This scattering problem is modeled by the Helmholtz system

$$
\begin{aligned}
-\Delta u^{\mathrm{tot}}-k^{2} u^{\mathrm{tot}} & =0 \text { in } \Omega^{+}, \\
\frac{\partial u^{\mathrm{tot}}}{\partial \hat{\mathbf{n}}} & =0 \text { on } \Gamma, \\
\lim _{|\mathbf{x}| \rightarrow \infty}|\mathbf{x}|\left(\frac{\partial u^{\mathrm{sca}}}{\partial|x|}-i k u^{\mathrm{sca}}\right) & =0
\end{aligned}
$$

where the last equation is the Sommerfeld radiation condition at infinity. Here, $u^{\text {sca }}$ denotes the scattered field, such that $u^{\text {tot }}=u^{\text {inc }}+u^{\text {sca }}$. The scatterer object is assumed to be impenetrable, hence $u^{\text {tot }}=0$ in $\Omega^{-}$.

Helmholtz problems are often solved with computational methods such as finitedifference, finite-element and spectral techniques. As opposed to these volumebased algorithms, we will use the surface-based BEM [40, 44, 41]. The basic idea behind the BEM is to reformulate the Helmholtz system into a boundary integral formulation and solve the scattering problem on the surface itself. In this chapter we will review the design of boundary integral equations with an emphasis on large-scale scattering problems at high frequencies. For this case, it is necessary to use modern matrix compression and preconditioning techniques. We will apply these state-of-the-art techniques to a challenging problem arising from medical high-intensity focused ultrasound simulations [25]. In [48] we have published an earlier version of some of the techniques presented in this chapter. There, more details about the engineering application can be found. Here, we give a more detailed analysis of the boundary integral formulations, include other formulations as well and explain the compression technique. Furthermore, this chapter uses a newer version of BEM++ which allows us to perform experiments on a larger scale.

The explicit use of the acoustic Green's function gives the BEM some major advantages compared to standard computational methods. First of all, the Sommerfeld radiation condition (1c) is exactly satisfied by boundary integral representations. There is thus no need for absorbing boundary conditions to artificially truncate the exterior region, as is required for volume-based discretization techniques [28]. This makes the BEM a natural choice for solving scattering problems in unbounded domains. Another positive effect from the Green's function is that well-chosen discretizations are essentially free of pollution and dispersion, even for low order discretizations using piecewise constant basis functions [29]. Furthermore, since the 
model equations live on the boundary only, surface meshes are being used. These are often easier to generate for complex geometries compared to volume meshes.

On the other hand, the BEM is not free of problems. For instance, it is crucial to carefully choose the correct type of boundary integral equation formulation. In particular for high-frequency problems it is necessary to choose a formulation that does not suffer from breakdown at certain resonant frequencies [1,2]. This will be the topic of Section 2.

In the case of large-scale simulations, the discrete system of equations is typically being solved with iterative linear solvers, which are asymptotically more efficient than direct solvers [3]. Furthermore, these methods mainly rely on matrix-vector multiplications, which are relatively easy to parallelize and for which acceleration algorithms are available. However, the required number of iterations can easily become prohibitively large for high-frequency problems, especially for the classical boundary integral formulations. In Section 3 we therefore review various operator preconditioning techniques for high-frequency applications and numerically assess their performance in Section 5.2.

A naive discretization of the boundary integral operators would lead to dense matrix problems and a complexity of $\mathscr{O}\left(N^{2}\right)$ for the assembly and the matrix-vector product, where $N$ is the number of elements. For a fixed number of surface elements per wavelength, i.e., $N \sim k^{2}$, the complexity will therefore scale as $\mathscr{O}\left(k^{4}\right)$. This is only feasible for small-scale problems. For large-scale applications it is vital to use acceleration schemes that reduce the computation time and memory footprint to realistic measures for present-day computer architectures. The most prominent of such methods are Fast Multiple Methods (FMM) [17, 16, 23] and hierarchical matrix techniques ( $\mathscr{H}$-matrices and their $\mathscr{H}^{2}$ and HSS variants) [32, 8, 6, 49, 35]. They achieve a complexity of $\mathscr{O}(N)$ or $\mathscr{O}(N \log (N))$ for the matrix-vector multiplication, depending on the frequency regime and the specific implementation. In Section 4 we will discuss the behavior of classical $\mathscr{H}$-matrix techniques for exterior scattering problems in more detail. While their complexity with respect to a growing wavenumber $k$ is asymptotically not as good as high-frequency FMM, they are kernel-independent, relatively easy to implement and offer good performance for a wide range of application relevant frequencies.

The numerical implementation of a high-frequency BEM is challenging, mainly because of the necessity of specialized acceleration techniques and quadrature rules for singular integral operators. In Section 5 we will introduce the open-source software library BEM++ [42] which has been used to perform all computational experiments in this chapter. This library was originally developed at University College London and provides a comprehensive Python toolbox to setup and solve Laplace, Helmholtz and Maxwell problems via the BEM. Matrix compression is integrated and various preconditioners are available for the efficient solution of large-scale problems. Fast computations are achieved because the core discretization and compression routines are written in $\mathrm{C}++$. All these routines are accessible via a highlevel Python interface, which provides a user-friendly programming environment. We will present code examples to demonstrate how, with only a limited amount of high-level instructions, an entire BEM simulation can be performed with BEM++. 
Tutorials in the form of IPython notebooks can be downloaded from the website of the BEM++ project (www.bempp.org).

Finally, in Section 6 we present the application of the fast BEM to a realistic problem arising from medical treatment planning in high-frequency focused ultrasound. The described problem will lead to a system with around half a million unknowns and simulates over one hundred wavelengths across the computational domain. This has been solved with BEM++ on a single workstation, thus confirming the capabilities of the efficient BEM presented in this chapter.

\section{Boundary integral formulations of high-frequency scattering}

In this section we review the standard combined field equations for boundary integral formulations of high-frequency scattering. Details and proofs of the statements given here can be found in standard textbooks such as [40, 44, 41]. A recent overview article of novel mathematical developments for high-frequency scattering formulations based on hybrid numerical-asymptotic methods is also given in [15]. While these hybrid numerical-asymptotic methods have the potential to solve scattering problems on certain geometries with an almost wavenumber independent convergence, they are not yet suitable for larger industrial applications with realistic meshes.

\subsection{Surface representation of the scattering model}

The reformulation of the exterior model into a surface model necessitates operators that map between the volume $\Omega^{-} \cup \Omega^{+}$and the boundary $\Gamma$. The map from the volume to the boundary is provided by the trace operators, which are denoted by $\gamma$. More specifically, the Dirichlet trace operators $\gamma_{0}^{-}$and $\gamma_{0}^{+}$are defined as the limit values of a field towards the interface from the interior and exterior domain, respectively, and the Neumann trace operators $\gamma_{1}^{-}$and $\gamma_{1}^{+}$are the corresponding normal derivatives. On the other hand, the potential operators map from the surface to the volume. They are defined as

$$
\begin{array}{ll}
(\mathscr{V} \psi)(\mathbf{x}):=\int_{\Gamma} G(\mathbf{x}, \mathbf{y}) \psi(\mathbf{y}) \mathrm{d} \Gamma(\mathbf{y}) & \text { for } \mathbf{x} \in \Omega^{-} \cup \Omega^{+}, \\
(\mathscr{K} \phi)(\mathbf{x}):=\int_{\Gamma} \partial_{n(\mathbf{y})} G(\mathbf{x}, \mathbf{y}) \phi(\mathbf{y}) \mathrm{d} \Gamma(\mathbf{y}) & \text { for } \mathbf{x} \in \Omega^{-} \cup \Omega^{+}
\end{array}
$$

and are called the single-layer and double-layer potential operators, respectively. Here, $\psi$ and $\phi$ denote surface potentials that live on the boundary only. The function $G(\mathbf{x}, \mathbf{y})$ is the acoustic Green's function defined by 


$$
G(\mathbf{x}, \mathbf{y}):=\frac{e^{i k|\mathbf{x}-\mathbf{y}|}}{4 \pi|\mathbf{x}-\mathbf{y}|} \quad \text { for } \mathbf{x} \neq \mathbf{y}
$$

and $\partial_{n(\mathbf{y})} G(\mathbf{x}, \mathbf{y})$ is its normal derivative along $\hat{\mathbf{n}}$ with respect to $\mathbf{y}$.

Using the single-layer and double-layer potential operator one can derive a representation formula for any radiating solution $u$ of the Helmholtz equation as

$$
u(\mathbf{x})=(\mathscr{V} \psi)(\mathbf{x})-(\mathscr{K} \phi)(\mathbf{x}) \quad \text { for } \mathbf{x} \in \Omega^{-} \cup \Omega^{+}
$$

with

$$
\begin{aligned}
& \psi=\gamma_{1}^{-} u-\gamma_{1}^{+} u, \\
& \phi=\gamma_{0}^{-} u-\gamma_{0}^{+} u
\end{aligned}
$$

being the jumps of the solution across the interface.

Taking the trace or normal derivative of both sides of the equality in Eq. (5) will result in an equation that is fully defined on the boundary. This necessitates the analysis of the traces and normal derivatives of potential operators. One can show that the following boundary operators are well defined almost everywhere if $\Gamma$ is piecewise smooth:

$$
\begin{aligned}
(V \psi)(\mathbf{x}) & :=\int_{\Gamma} G(\mathbf{x}, \mathbf{y}) \psi(\mathbf{y}) \mathrm{d} \Gamma(\mathbf{y}) & & \text { for } \mathbf{x} \in \Gamma, \\
(K \phi)(\mathbf{x}) & :=\int_{\Gamma} \partial_{n(\mathbf{y})} G(\mathbf{x}, \mathbf{y}) \phi(\mathbf{y}) \mathrm{d} \Gamma(\mathbf{y}) & & \text { for } \mathbf{x} \in \Gamma, \\
(T \psi)(\mathbf{x}) & :=\int_{\Gamma} \partial_{n(\mathbf{x})} G(\mathbf{x}, \mathbf{y}) \psi(\mathbf{y}) \mathrm{d} \Gamma(\mathbf{y}) & & \text { for } \mathbf{x} \in \Gamma, \\
(D \phi)(\mathbf{x}) & :=-\partial_{n(\mathbf{x})} \int_{\Gamma} \partial_{n(\mathbf{y})} G(\mathbf{x}, \mathbf{y}) \phi(\mathbf{y}) \mathrm{d} \Gamma(\mathbf{y}) & & \text { for } \mathbf{x} \in \Gamma .
\end{aligned}
$$

Moreover, for piecewise smooth $\Gamma$ the following jump relations are defined almost everywhere:

$$
\begin{aligned}
V \psi & =\gamma_{0}^{-}(\mathscr{V} \psi)=\gamma_{0}^{+}(\mathscr{V} \psi), \\
K \phi & =\gamma_{0}^{-}(\mathscr{K} \phi)+\frac{1}{2} \phi=\gamma_{0}^{+}(\mathscr{K} \phi)-\frac{1}{2} \phi, \\
T \psi & =\gamma_{1}^{-}(\mathscr{V} \psi)-\frac{1}{2} \psi=\gamma_{1}^{+}(\mathscr{V} \psi)+\frac{1}{2} \psi, \\
D \phi & =-\gamma_{1}^{-}(\mathscr{K} \phi)=-\gamma_{1}^{+}(\mathscr{K} \phi) .
\end{aligned}
$$

For the precise definition in the general Lipschitz case see e.g. [44, Chapter 6].

The operators $V, K, T$, and $D$ are called the single-layer, double-layer, adjoint double-layer and hypersingular boundary integral operator, respectively, and satisfy the mapping properties 


$$
\begin{array}{rlrl}
V: \mathscr{H}^{-\frac{1}{2}}(\Gamma) & \rightarrow \mathscr{H}^{\frac{1}{2}}(\Gamma), & K: \mathscr{H}^{\frac{1}{2}}(\Gamma) \rightarrow \mathscr{H}^{\frac{1}{2}}(\Gamma), \\
T: \mathscr{H}^{-\frac{1}{2}}(\Gamma) \rightarrow \mathscr{H}^{-\frac{1}{2}}(\Gamma), & D: \mathscr{H}^{\frac{1}{2}}(\Gamma) \rightarrow \mathscr{H}^{-\frac{1}{2}}(\Gamma)
\end{array}
$$

for fractional Sobolev spaces $\mathscr{H}^{\frac{1}{2}}(\Gamma)$ and $\mathscr{H}^{-\frac{1}{2}}(\Gamma)$. In addition, the identity boundary operator is denoted by $I$. Boundary integral equations can now readily be derived by taking traces of representation formulas. The simplest forms are based on the normal derivative of the single-layer or double-layer potential operator only. Drawback of these operators is their nontrivial nullspace at resonant frequencies. An effective approach to mitigate the breakdown at resonances is to consider combined field integral equations that are uniquely solvable for all real wavenumbers.

\subsection{The Burton-Miller combined boundary integral equation}

A classical combined field integral equation for the scattering problem (1) is the Burton-Miler formulation [13]. This formulation is free of spurious resonances and the unique solution has a direct interpretation as the trace of the exterior total field on the boundary $\Gamma$. We start with the direct representation (5) of the scattered field, i.e., $u^{\text {sca }}=\mathscr{V} \psi-\mathscr{K} \phi$ where the surface potentials $\psi$ and $\phi$ are given by the jumps of the scattered field across the boundary and can be simplified as

$$
\begin{aligned}
& \psi=\gamma_{1}^{-} u^{\mathrm{sca}}-\gamma_{1}^{+} u^{\mathrm{sca}}=\gamma_{1}^{-}\left(u^{\mathrm{tot}}-u^{\mathrm{inc}}\right)+\gamma_{1}^{+} u^{\mathrm{inc}}=0, \\
& \phi=\gamma_{0}^{-} u^{\mathrm{sca}}-\gamma_{0}^{+} u^{\mathrm{sca}}=\gamma_{0}^{-}\left(u^{\mathrm{tot}}-u^{\mathrm{inc}}\right)-\gamma_{0}^{+}\left(u^{\mathrm{tot}}-u^{\mathrm{inc}}\right)=-\gamma_{0}^{+} u^{\mathrm{tot}}
\end{aligned}
$$

because the total field is zero in the interior and the incident wave field smooth across the boundary. This reduces the representation formula to

$$
u^{\mathrm{sca}}=\mathscr{K}(\varphi), \quad \varphi=\gamma_{0}^{+} u^{\mathrm{tot}} .
$$

Taking the exterior Neumann trace $\gamma_{1}^{+}$of this representation formula yields

$$
-\gamma_{1}^{+} u^{\text {inc }}=-D \varphi
$$

where the boundary condition and jump relation (14) have been used. The interior Dirichlet trace $\gamma_{0}^{-}$of the representation formula results in

$$
-\gamma_{0}^{+} u^{\mathrm{inc}}=K \varphi-\frac{1}{2} \varphi
$$

where the zero interior field, jump relation (12) and smoothness of the incident wave field have been used.

Both boundary integral equations (16) and (17) solve the scattering problem for the same surface potential. Any linear combination will therefore solve the scattering problem as well. That is, for a coupling parameter $\eta \in \mathbb{C}$, the Burton-Miller 
formulation

$$
A_{\eta} \varphi=u^{\mathrm{inc}}+\eta \partial_{n} u^{\mathrm{inc}}
$$

with

$$
A_{\eta}:=\left(\frac{1}{2} I-K\right) \varphi+\eta D \varphi
$$

solves the scattering problem with the representation formula (15). The BurtonMiller formulation is uniquely solvable for $\mathfrak{I}(\eta) \neq 0$ and $\eta=i / k$ is a good choice of coupling parameter [37].

\subsection{Regularizing the Burton-Miller formulation}

We notice that the Burton-Miller formulation (18) is not without problems. The operator $\left(\frac{1}{2} I-K\right)$ is minus the interior trace of the double layer potential operator $\mathscr{K}$ and maps from $\mathscr{H}^{\frac{1}{2}}(\Gamma)$ into $\mathscr{H}^{\frac{1}{2}}(\Gamma)$, whereas the hypersingular operator $D$ maps from $\mathscr{H}^{\frac{1}{2}}(\Gamma)$ into $\mathscr{H}^{-\frac{1}{2}}(\Gamma)$. A solution to this mismatch in mapping characteristics is to consider regularized combined field operators [12]. For a regularization operator

$$
\mathscr{R}: \mathscr{H}^{-\frac{1}{2}}(\Gamma) \rightarrow \mathscr{H}^{\frac{1}{2}}(\Gamma),
$$

the regularized Burton-Miller formulation reads

$$
\left(\frac{1}{2} I-K\right) \varphi+\mathscr{R} D \varphi=u^{\mathrm{inc}}+\mathscr{R} \partial_{n} u^{\mathrm{inc}},
$$

where now the operator $A_{\mathscr{R}}:=\left(\frac{1}{2} I-K\right)+\mathscr{R} D$ is well defined on $\mathscr{H}^{\frac{1}{2}}(\Gamma)$. The design of sophisticated regularization techniques forms the basis of the efficient preconditioning strategies discussed in Section 3.

\subsection{Indirect formulations}

An alternative approach to obtaining a combined field integral equation for the scattering problem (1) is to use an indirect representation of the scattered field as the linear combination

$$
u^{\mathrm{sca}}=-i \mu \mathscr{V} \phi+\mathscr{K}(\mathscr{R} \phi)
$$

where regularization with $\mathscr{R}$ has been applied. Taking the exterior Neumann trace $\gamma_{1}^{+}$ on both sides and using $\partial_{n} u^{\mathrm{inc}}=-\partial_{n} u^{\mathrm{sca}}$ on boundary $\Gamma$ results in

$$
-\partial_{n} u^{\mathrm{inc}}=i \mu\left(\frac{1}{2} I-T\right) \phi-D(\mathscr{R} \phi) .
$$

Traditionally, equation (21) without the regularization is called the BrakhageWerner formulation [9]. In [11] it is suggested to use $\mu=1$ for high-frequency scattering problems. 


\subsection{Boundary element methods}

For the discretization of boundary integral operators typically either collocation or Galerkin methods are used. While collocation methods are easier to implement, the Galerkin method has advantages with respect to coupling with finite element methods, symmetry of the resulting operators, and assembly on non-smooth domains. Here, we focus on Galerkin methods for the Burton-Miller formulation (18).

Let $\Gamma_{h}$ be a triangulation of $\Gamma$ with $n$ nodes $\hat{\mathbf{x}}_{j}, j=1, \ldots, n$. Let $\phi_{j}$ be a continuous piecewise linear function defined on $\Gamma_{h}$ such that $\phi_{j}\left(\hat{\mathbf{x}}_{i}\right)=\left\{\begin{array}{l}1, i=j \\ 0, i \neq j\end{array}\right.$. Let us denote by $V_{h}:=\left\{\sum_{j=1}^{n} v_{j} \phi_{j}, v_{j} \in \mathbb{C}\right\}$ the space spanned by the nodal basis functions $\phi_{j}$. Define the standard real dual pairing

$$
\langle\varphi, \vartheta\rangle:=\int_{\Gamma} \varphi(\mathbf{y}) \cdot \vartheta(\mathbf{y}) \mathrm{d} \Gamma(\mathbf{y})
$$

The Galerkin discretization of the Burton-Miller formulation is now given as the discrete matrix problem

$$
\bar{A}_{\eta} \mathbf{v}=\mathbf{b}
$$

with $\left[\bar{A}_{\eta}\right]_{i j}=\left\langle A_{\eta} \phi_{j}, \phi_{i}\right\rangle$ and $b_{i}=\left\langle u^{\text {inc }}, \phi_{i}\right\rangle+\left\langle\eta \partial_{n} u^{\text {inc }}, \phi_{i}\right\rangle$.

The matrix $\bar{A}_{\eta}$ is given as $\bar{A}_{\eta}=\frac{1}{2} \bar{I}-\bar{K}+\eta \bar{D}$, where the individual matrix entries are computed as

$$
\begin{aligned}
{[\bar{I}]_{i j}=} & \int_{\Gamma} \phi_{i}(\mathbf{x}) \phi_{j}(\mathbf{x}) \mathrm{d} \Gamma(\mathbf{x}) \\
{[\bar{K}]_{i j}=} & \int_{\Gamma} \phi_{i}(\mathbf{x}) \int_{\Gamma} \partial_{n(\mathbf{y})} G(\mathbf{x}, \mathbf{y}) \phi_{j}(\mathbf{y}) \mathrm{d} \Gamma(\mathbf{y}) \mathrm{d} \Gamma(\mathbf{x}) \\
{[\bar{D}]_{i j}=} & -\int_{\Gamma} \phi_{i}(\mathbf{x}) \partial_{n(\mathbf{x})} \int_{\Gamma} \partial_{n(\mathbf{y})} G(\mathbf{x}, \mathbf{y}) \phi(\mathbf{y}) \mathrm{d} \Gamma(\mathbf{y}) \mathrm{d} \Gamma(\mathbf{x}) \\
= & \int_{\Gamma} \int_{\Gamma} G(\mathbf{x}, \mathbf{y})\left(\operatorname{curl}_{\Gamma} \phi_{i}(\mathbf{x}) \cdot \operatorname{curl}_{\Gamma} \phi_{j}(\mathbf{y})\right) \mathrm{d} \Gamma(\mathbf{y}) \mathrm{d} \Gamma(\mathbf{x}) \\
& -k^{2} \int_{\Gamma} \int_{\Gamma} G(\mathbf{x}, \mathbf{y}) \phi_{i}(\mathbf{x}) \phi_{j}(\mathbf{y})(\hat{\mathbf{n}}(\mathbf{x}) \cdot \hat{\mathbf{n}}(\mathbf{y})) \mathrm{d} \Gamma(\mathbf{y}) \mathrm{d} \Gamma(\mathbf{x})
\end{aligned}
$$

For the hypersingular operator $D$ the last formula follows from integration by parts and leads to a weakly singular integral. We also note that $\bar{D}^{T}=\bar{D}$ and $\bar{K}^{T}=\bar{T}$, where $\bar{T}$ is the discretization of the adjoint double-layer boundary operator.

Evaluating these integrals requires singularity-adapted quadrature rules. A general fully numerical quadrature scheme based on regularizing coordinate transformations is described in [41]. However, this scheme can still lead to large errors in situations such as sharp edges, two parallel triangles that are close to each other, and almost degenerate triangles. Alternative quadrature schemes that can deal with some of these issues are described for example in [38]. 
If instead of a scalar $\eta$ we use a regularizing operator $\mathscr{R}$, then the operator $A_{\mathscr{R}}$ is well defined on $\mathscr{H}^{\frac{1}{2}}(\Gamma)$ and we can formulate a variational problem to find $\phi \in$ $\mathscr{H}^{\frac{1}{2}}(\Gamma)$ such that

$$
\left\langle A_{\mathscr{R}} \phi, \vartheta\right\rangle=\left\langle u^{\mathrm{inc}}, \vartheta\right\rangle+\left\langle\mathscr{R} \partial_{n} u^{\mathrm{inc}}, \vartheta\right\rangle, \quad \forall \vartheta \in \mathscr{H}^{-\frac{1}{2}}(\Gamma),
$$

where we now interpret the dual pairing $\langle\cdot, \cdot\rangle$ as a dual pairing on $\mathscr{H}^{\frac{1}{2}}(\Gamma) \times$ $\mathscr{H}^{-\frac{1}{2}}(\Gamma)$. The corresponding discrete left-hand-side matrix is then given as

$$
\overline{A_{\mathscr{R}}}:=\frac{1}{2} \bar{I}-\bar{K}+\overline{\mathscr{R}} \bar{I}^{-1} \bar{D}
$$

where $[\mathscr{R}]_{i j}=\left\langle\mathscr{R} \phi_{j}, \phi_{i}\right\rangle$. To analyze the Galerkin variational formulation, techniques as discussed in [12] can now be used.

The discretization above uses the same space $V_{h}$ of continuous piecewise linear nodal basis functions to discretize $\mathscr{H}^{\frac{1}{2}}(\Gamma)$ and $\mathscr{H}^{-\frac{1}{2}}(\Gamma)$. However, we use the space $\mathscr{H}^{-\frac{1}{2}}(\Gamma)$ to represent Neumann data. Hence, this approximation is only suitable if the boundary $\Gamma$ is sufficiently smooth to support continuous Neumann data. For more general Lipschitz domains we can expect discontinuities and a more natural basis of $\mathscr{H}^{-\frac{1}{2}}(\Gamma)$ is a space of discontinuous piecewise constant functions. A stable dual pairing between continuous nodal basis functions and a space of piecewise constant discontinuous functions can be achieved by defining the discontinuous functions on the dual grid [33].

\section{Operator preconditioners for high-frequency problems}

The classical Burton-Miller formulation suffers from poor convergence for highfrequency problems on general domains. The main reason is that the hypersingular operator $D$ acts like an unbounded differential operator from $\mathscr{H}^{\frac{1}{2}}(\Gamma)$ to $\mathscr{H}^{-\frac{1}{2}}(\Gamma)$. As explained in Section 2.3, including a regularization operator fixes the mismatch in function spaces. Being an operator preconditioner, this regularization should be carefully chosen such that it improves the conditioning of the discrete system [43, $34,36]$. In practice, the regularization is ideally designed such that the resulting boundary integral operator is a compact perturbation of the identity operator.

In this section we will focus on two types of regularization, based on a highfrequency approximation of the Neumann-to-Dirichlet $(\mathrm{NtD})$ map and the singlelayer boundary operator. These operator preconditioners do not depend on the discretization method and can readily be combined with acceleration schemes such as $\mathscr{H}$-matrix compression. 


\subsection{OSRC preconditioning}

The On-Surface Radiation Condition (OSRC) preconditioner is based on the idea of finding a local surface approximation of the NtD map [4, 5, 20]. For $\vartheta \in \mathscr{H}^{-\frac{1}{2}}(\Gamma)$ we define the exterior Neumann-to-Dirichlet map $N_{\mathrm{ex}}^{+}: \mathscr{H}^{-\frac{1}{2}}(\Gamma) \rightarrow \mathscr{H}^{\frac{1}{2}}(\Gamma)$ as $N_{\mathrm{ex}}^{+}(\vartheta):=\gamma_{0}^{+} u_{\vartheta}$, where $u_{\vartheta}$ is the solution of the exterior Helmholtz problem

$$
\begin{aligned}
-\Delta u_{\vartheta}-k^{2} u_{\vartheta} & =0 \text { in } \Omega^{+}, \\
\frac{\partial u_{\vartheta}}{\partial \hat{\mathbf{n}}} & =\vartheta \text { on } \Gamma, \\
\lim _{|\mathbf{x}| \rightarrow \infty}|\mathbf{x}|\left(\frac{\partial u_{\vartheta}}{\partial|x|}-i k u_{\vartheta}\right) & =0 .
\end{aligned}
$$

Using the NtD map it follows from the exterior Calderón projector [44, Section 7.5] that

$$
\left(\frac{1}{2} I-T-D N_{\mathrm{ex}}^{+}\right) \vartheta=\vartheta
$$

for $\vartheta \in \mathscr{H}^{-\frac{1}{2}}(\Gamma)$. Assume that an approximation $\widetilde{N_{\mathrm{ex}}^{+}}$of the NtD map is given. Then, after discretization, we obtain

$$
\left(\frac{1}{2} \bar{I}-\bar{T}-\bar{D} \bar{I}^{-1} \overline{\widetilde{N_{\mathrm{ex}}^{+}}}\right) v \approx \bar{I} v
$$

Notice that since $\bar{T}^{T}=\bar{K}$ and $\bar{D}^{T}=\bar{D}$ the transpose of the left-hand-side operator equals the regularized Burton-Miller operator with $\overline{\mathscr{R}}^{T}=-\overline{\widetilde{N_{\mathrm{ex}}^{+}}}$. This shows that a good approximation to the NtD map results in an excellent preconditioner.

Unfortunately, the NtD map is a non-local pseudo-differential operator whose computation itself involves the solution of an exterior Helmholtz problem which makes its direct use as preconditioner impractical. However, there are efficient approximations that can be used. We have already encountered the most basic approximation, namely $N_{\mathrm{ex}}^{+} \approx \frac{1}{i k}$ giving the classical Burton-Miller operator with $\eta=i / k$. Alternatively, a more accurate approximation of the NtD map can be derived as

$$
N^{o s r c}=\frac{1}{i k}\left(1+\frac{\Delta_{\Gamma}}{k_{\varepsilon}^{2}}\right)^{-1 / 2}
$$

where $\Delta_{\Gamma}$ denotes the surface Laplace-Beltrami operator [4, 5]. The occurrence of singularities is prevented with the use of a damped wavenumber $k_{\varepsilon}=k(1+i \varepsilon)$. Based on a spectral analysis on a sphere, a good choice of damping is $\varepsilon=$ $0.4(k R)^{-2 / 3}$ with $R$ the radius of the object [20]. Localization of this operator is achieved with a Padé approximation of size $n$ and a nonzero branch cut, typically 4 and $\pi / 3$, respectively. The application of the OSRC operator is now reduced to solving a set of $(n+1)$ surface Helmholtz equations with complex-valued wavenumber. 
The solution procedure of these local operators can efficiently be performed with sparse LU-factorization.

The OSRC-preconditioned Burton-Miller formulation

$$
\left(\frac{1}{2} I-K\right) \varphi-N^{o s r c} D \varphi=u^{\text {inc }}-N^{o s r c} \partial_{n} u^{\text {inc }}
$$

is uniquely solvable in $\mathscr{H}^{\frac{1}{2}}(\Gamma)$ on a smooth surface, for any wavenumber and nonzero damping factor [20]. Moreover, the boundary integral operator reduces to

$$
\left(\frac{1}{2} I-K\right) \varphi-N^{o s r c} D \varphi=\left(\frac{1}{2}+\frac{k_{\varepsilon}}{2 k}\right) I+C
$$

for a compact operator $C$ if $\Gamma$ is sufficiently smooth. This is a second kind Fredholm integral equation and has a clustering of eigenvalues, resulting in fast convergence of linear solvers.

\subsection{Regularization by single-layer boundary operators}

Another strategy to achieve regularization of the hypersingular operator is to consider the single-layer potential. With Calderón identities [44, Corollary 6.19], one can show that

$$
\begin{aligned}
& D V=\frac{1}{4} I-T^{2}, \\
& V D=\frac{1}{4} I-K^{2} .
\end{aligned}
$$

Hence, if $\Gamma$ is sufficiently smooth, then the product of the single-layer and the hypersingular boundary operator is a compact perturbation of a scaled identity. However, the single-layer operator alone is not a good choice of a regularizer due to the existence of resonances. A solution was proposed in [11], where the single-layer boundary operator $V_{\kappa}$ with wavenumber $\kappa=i k / 2$ was investigated as regularizer for the Brakhage-Werner formulation (21). Specifically,

$$
i\left(\frac{1}{2} I-T\right) \varphi-D V_{\kappa} \varphi=-\partial_{n} u^{\mathrm{inc}}
$$

for a coupling parameter $\mu=1$. Similarly, this regularization can also be applied to the Burton-Miller formulation (19). For sufficiently smooth $\Gamma$ this formulation is again a perturbation of a scaled identity because $V_{\kappa} D=(V+C) D$, where $C$ is a compact operator [12, Lemma 2.1] and $V$ is the single-layer operator for the original wavenumber $k$. The imaginary-wavenumber single-layer operator can be evaluated relatively cheap as it allows a very efficient low-rank representation. 

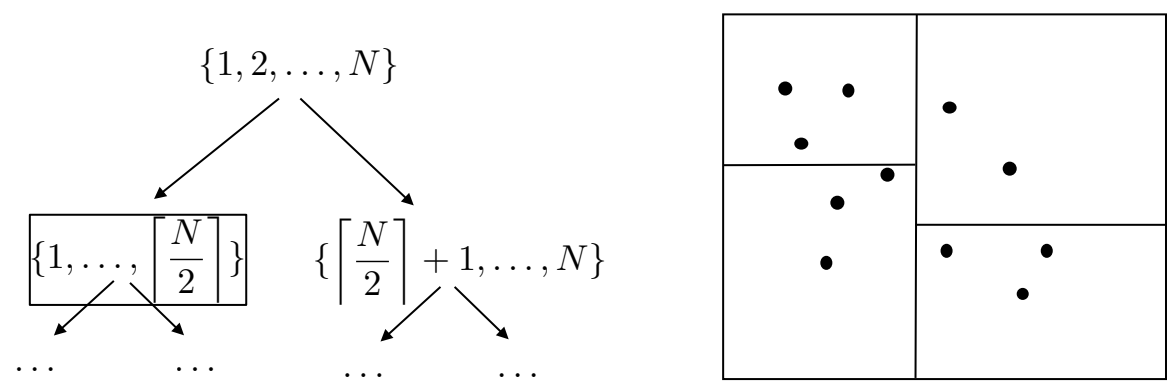

Fig. 1 Division of degrees of freedom into a cluster tree.

\section{Fast $\mathscr{H}$-matrix assembly}

Hierarchical ( $\mathscr{H}$-)matrix compression based on adaptive cross approximation (ACA) is a widely used technique to assemble boundary integral operators in a compressed format. It has a complexity of $\mathscr{O}(N \log N)$ for compression and evaluation of matrixvector products, where $N$ denotes the number of global degrees of freedom. This approach is relatively easy to implement, easily parallelizable, and builds a direct algebraic representation of the compressed operator that allows very fast matrix-vector products, compared to FMM. Main disadvantages are the longer setup time and often significantly higher memory consumption than FMM. However, particularly for low-frequency or non-oscillatory problems the performance is often excellent. Moreover, even though standard $\mathscr{H}$-matrix compression does not scale well asymptotically as $k \rightarrow \infty$, its practical performance even for higher-frequency problems is often very good as we will see in this and the following sections.

\subsection{The fundamentals of $\mathscr{H}$-matrix compression}

In this section we give a brief overview of the main ideas of $\mathscr{H}$-matrix compression. More details can be found in [7, 32]. The $\mathscr{H}$-matrix compression is based on a geometric subdivision of the set of degrees of freedom (dofs) $I$ in the boundary element mesh into a cluster tree $T(I)$. On each level the dofs are subdivided into two geometrically separated sets, as depicted in Fig. 1. The leafs of the cluster tree are reached when the number of dofs in each subdivision is below a specified tolerance. Given a set of dofs $I$ for the test functions and a set of dofs $J$ for the basis functions in the BEM discretization a block cluster tree $T(I \times J)$ is now constructed as follows.

1. The root of the block cluster tree is the product index set $b_{0}=\tau \times \sigma$ with $\tau=I$ and $\sigma=J$.

2. Given a node $b^{\prime}=\tau^{\prime} \times \sigma^{\prime}$ of the block cluster tree, where $\tau^{\prime}$ and $\sigma^{\prime}$ are nodes of the corresponding cluster trees $T(I)$ and $T(J)$ : 
- Stop the recursion if the current node satisfies an admissibility condition or if one of the cluster tree nodes $\sigma^{\prime}$ and $\tau^{\prime}$ is a leaf node.

- If the recursion is not stopped, define the sons of the block cluster tree node $b^{\prime}$ as the set $\left\{\tau_{1}^{\prime} \times \sigma_{1}^{\prime}, \tau_{1}^{\prime} \times \sigma_{2}^{\prime}, \tau_{2}^{\prime} \times \sigma_{1}^{\prime}, \tau_{2}^{\prime} \times \sigma_{2}^{\prime}\right\}$ for the sons $\tau_{i}^{\prime}$ and $\sigma_{j}^{\prime}, i, j=1,2$ of the cluster tree nodes $\tau^{\prime}$ and $\sigma^{\prime}$.

The admissibility condition is satisfied if the geometric bounding boxes $X$ and $Y$ associated with the cluster nodes $\tau^{\prime}$ and $\sigma^{\prime}$ satisfy a separability condition. A frequently used condition is given as

$$
\min \{\operatorname{diam}(X), \operatorname{diam}(Y)\} \leq \alpha \operatorname{dist}(X, Y) .
$$

Here, diam denotes the diameter of a bounding box and dist the distance of two bounding boxes. The parameter $\alpha$ controls how strongly separated $X$ and $Y$ must be so that the admissibility condition is satisfied. By default, BEM++ uses a weaker condition given as

$$
\operatorname{dist}(\mathrm{X}, \mathrm{Y})>0 .
$$

This works sufficiently well in practice and usually leads to a fewer number of blocks on the block cluster tree.

Once the generation of the block cluster tree has been completed, a compressed representation of the BEM matrix $\bar{A}$ can be assembled as follows. Let $b^{\prime}=\tau^{\prime} \times \sigma^{\prime} \in$ $\mathscr{L}(T(I \times J))$, the set of all leaf blocks of the block cluster tree $T(I \times J)$.

- If $b^{\prime}$ is not admissible, then evaluate all entries of $\bar{A}_{\tau^{\prime} \times \sigma^{\prime}}$, the restriction of $\bar{A}$ onto the index set $\tau^{\prime} \times \sigma^{\prime}$, directly and store the corresponding dense representation.

- If $b^{\prime}$ is admissible, then store a low rank representation $\bar{A}_{\tau^{\prime} \times \sigma^{\prime}} \approx U_{b^{\prime}} \times V_{b^{\prime}}^{H}$, where $U_{b^{\prime}}$ is of dimension $\left|\tau^{\prime}\right| \times t$ and $V_{b^{\prime}}$ is of dimension $\left|\sigma^{\prime}\right| \times t$ where $t$ denotes the local rank.

To obtain a low-rank representation, a frequently used algorithm is Adaptive Cross Approximation (ACA). It is a heuristic algorithm that often works remarkably well and allows an approximate error control to determine the local rank $t$ adaptively given a global error bound. However, most importantly, ACA only needs to compute a small fraction of the elements of the original matrix so that even very large BEM discretizations can be assembled on standard workstation systems.

Finally, often the above described compression procedure is intermixed with a recompression scheme in which after the compression of individual son blocks of a block cluster tree node $b^{\prime}$ a compression of $b^{\prime}$ itself is attempted using information from the sons. If this needs less memory than the original son representations, then the low-rank compression of $b^{\prime}$ itself is used instead and the sons deleted.

\subsection{The $\mathscr{H}$-matrix compression at high frequencies}

The above described compression scheme is very efficient for low or non-oscillatory problems. However, for high-frequency problems the minimum rank required in 
each admissible block grows with the wavenumber. Let us consider the block cluster leaf node $b^{\prime}=\tau^{\prime} \times \sigma^{\prime}$ and the corresponding bounding boxes $X$ and $Y$. Given the Green's function $G(\mathbf{x}, \mathbf{y})$, the efficiency of the above described $\mathscr{H}$-matrix compression depends on the number $t_{\varepsilon}$, such that

$$
\left\|G(\mathbf{x}, \mathbf{y})-\sum_{j=1}^{t_{\varepsilon}} g_{j}(\mathbf{x}) h_{j}(\mathbf{y})\right\|_{X \times Y}<\varepsilon
$$

for given $\varepsilon$. The number $t_{\varepsilon}$ is the minimum number of terms needed for a low-rank representation of the Green's function with accuracy $\varepsilon$. In [22] it is shown that

$$
k^{2-\delta} \lesssim t_{\varepsilon} \lesssim k^{2+\delta}, \quad \forall \delta>0
$$

The overall computational cost of compression and evaluation is linear with respect to the rank estimate $t$ in the admissible blocks, that is, the complexity scales like $\mathscr{O}(t N \log N)$. However, the rank $t$ is dependent on $N$ in high-frequency scattering. We typically choose a fixed number of dofs per wavelength, that is $N \sim k^{2}$. Together with (28) it therefore follows that $t \sim N$ giving an overall asymptotic complexity of $\mathscr{O}\left(N^{2} \log N\right)$ for $\mathscr{H}$-matrix compression. This would make $\mathscr{H}$-matrices unfeasible for large-scale problems in the limit $k \rightarrow \infty$.

Fortunately, in practice the behavior seems much better for realistic wavenumbers. In Table 1 we show performance results for the compression of the standard single-layer boundary operator $V$ with piecewise constant basis functions on the unit sphere for varying wavenumbers. We discretize the sphere with around 10 elements per wavelength, that is, $h=2 \pi /(10 k)$. For the ACA we choose an error tolerance of $10^{-5}$, which is sufficient for a wide range of applications. The timing results were done on a 20 cores, two processor Intel Xeon E5-2670 workstation with $2.5 \mathrm{Ghz}$ and 192 GB RAM. The compression rate measures how much memory the $\mathscr{H}$-matrix requires compared to a dense matrix of the same size. Recompression was not enabled. Also, BEM++ currently ignores the symmetry of the single-layer boundary operator, which could give another factor two saving. For the highest wavenumber $k=80$ with 480 thousand elements the assembly time is roughly 7.8 minutes and the memory consumption is $62 \mathrm{~GB}$.

It is interesting to measure the growth rate of the memory in dependence on $N$. We assume a memory growth of $\mathscr{O}\left(N^{\beta}\right)$ for some $\beta>0$. The last column in Table 1 shows estimates for $\beta$ by comparing the memory growth from one wavenumber to the next. The effective exponent is around 1.3 , which is significantly better than the asymptotic worst-case consideration given above and makes it possible to apply $\mathscr{H}$-matrices to many realistic high-frequency problems. 


\begin{tabular}{c|c|c|c|c|c}
$k$ & $N$ & memory (Mb) & compression (\%) & time (sec) & Growth rate $\beta$ \\
\hline 1 & 114 & 0.19 & 94.6 & $8.3 \mathrm{E}-2$ & - \\
5 & 2136 & 39.6 & 56.9 & 0.53 & 1.83 \\
10 & 7832 & 255 & 27.3 & 2.29 & 1.43 \\
20 & 30404 & $1.62 \mathrm{E} 3$ & 11.5 & 16.6 & 1.36 \\
30 & 68078 & $4.75 \mathrm{E} 3$ & 6.71 & 36.6 & 1.34 \\
40 & 120500 & $1.03 \mathrm{E} 4$ & 4.63 & 72.4 & 1.35 \\
50 & 188146 & $1.84 \mathrm{E} 4$ & 3.41 & $1.3 \mathrm{E} 2$ & 1.30 \\
60 & 270276 & $2.99 \mathrm{E} 4$ & 2.68 & $2.05 \mathrm{E} 2$ & 1.33 \\
70 & 367276 & $4.44 \mathrm{E} 4$ & 2.16 & $3.22 \mathrm{E} 2$ & 1.30 \\
80 & 480024 & $6.37 \mathrm{E} 4$ & 1.81 & $4.67 \mathrm{E} 2$ & 1.34
\end{tabular}

Table 1 The performance of the $\mathscr{H}$-matrix compression of the single-layer boundary operator $V$ on the unit sphere with varying wavenumber.

\subsection{Modern developments}

The standard $\mathscr{H}$-matrix approximations are popular for many applications because of their ease of implementation and relatively good performance. However, recent FMM developments can significantly outperform classical $\mathscr{H}$-matrix techniques. While FMM uses hierarchical basis information to propagate information from the sources to the targets this is not the case for $\mathscr{H}$-matrices. A remedy for this is given by $\mathscr{H}^{2}$-matrices [8]. These are algebraically equivalent to FMM and refine the $\mathscr{H}$ matrix format by exploiting hierarchical information within the cluster bases. This reduces the complexity of compression and matrix-vector product for low-frequency problems to $\mathscr{O}(N)$ instead of $\mathscr{O}(N \log N)$. A novel development specifically for highfrequency problems are wideband $\mathscr{H}$-matrix techniques. They exploit that within a cone of opening angle $\theta \sim \frac{1}{k}$ the source and target clusters admit low-rank representations even for large wavenumber [23]. The difficulty is that these novel wideband $\mathscr{H}$-matrix approaches need to deal with a very large number of small block clusters. The implementation in [6] uses a mixture of $\mathscr{H}$-matrix approximations for the near-field and $\mathscr{H}^{2}$-matrix approximations for the far-field to efficiently deal with this large number of block clusters.

\section{High-frequency boundary element simulations with BEM++}

Boundary integral formulations can conveniently be implemented with the opensource library BEM++ [42]. As will be shown in this section, only high-level instructions are necessary to perform a BEM simulation. Apart from the code snippets in this section, an IPython notebook of the OSRC-preconditioned Burton-Miller formulation can be downloaded from the BEM++ website (www.bempp.org). 


\subsection{Creating and solving an OSRC-preconditioned Burton-Miller formulation}

In the following we will describe the implementation and solution of the OSRCpreconditioned Burton-Miller formulation for the scattering of a plane wave incident field

$$
u^{\text {inc }}(x, y, z)=e^{i k x}
$$

which travels in the $x$-direction.

The BEM++ framework can be used as a Python library, imported with the usual command.

import bempp.api

The first step for the implementation of a boundary element simulation is to specify the model data such as incident wave field and scatterer object. In this example we specify the incident field by defining a corresponding Python function. Other ways of specifying boundary data are also possible.

A Python function that specifies an incident field takes as input arguments the location $\mathrm{x}$, normal direction $\mathrm{n}$, and optionally the region domain_index of the object. The following two functions specify the incident field and its normal derivative. The NumPy array result stores the value of the function in each dimension.

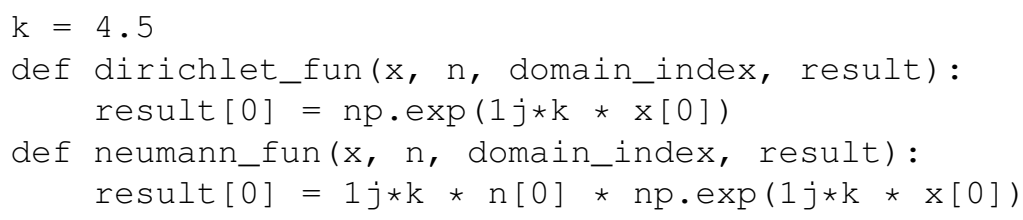

Several canonical objects can readily be created with $\mathrm{BEM}++$, such as a sphere, cube and ellipsoid. Optionally, the mesh size h can be passed, e.g. to guarantee an oversampling of ten elements per wavelength. The import of arbitrary triangular surface meshes in Gmsh format [27] is also possible. Alternatively, the node and connectivity information of a mesh can be specified. In the following we define the mesh of an ellipsoid with radius 3 in the $x$-direction and 1 in the other directions.

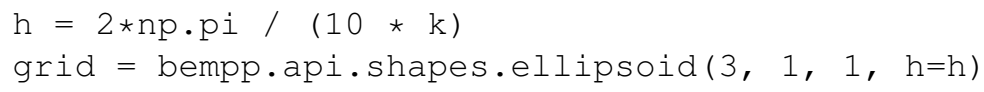

As finite element space, the BEM++ library provides continuous and discontinuous polynomial function spaces up to high-order and also function spaces defined on the barycentric mesh. Here, we only need the standard P1-elements.

space = bempp.api.function_space(grid, ' $\mathrm{P}^{\prime}, 1$ )

The native BEM++ object GridFunction provides functionality to store boundary data of the wave fields and also projections of the excitation field onto the boundary element space. 


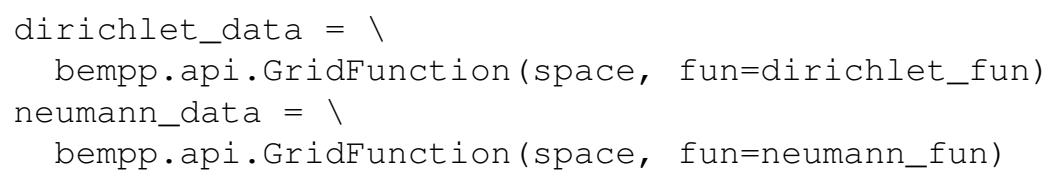

The creation of the boundary integral operators requires the specification of the mapping properties on the boundary element spaces, i.e., the domain, range and dual-to-range (test) space. For Galerkin discretization only the domain and the test space are required. The range space allows the implementation of an operator algebra that automatically creates the correct mass matrix transformations. This will be needed in the following. The OSRC-approximated NtD operator only requires one space object associated with a space of continuous functions to discretize the underlying Laplace-Beltrami operator, where it is always assumed that the domain, range and dual to range space are identical.

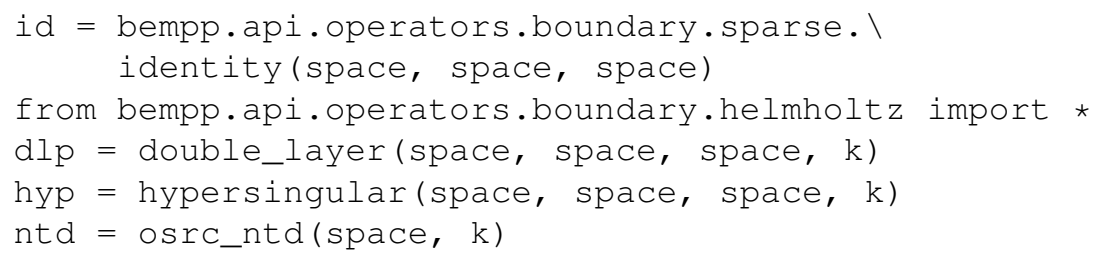

The created boundary integral operators are abstract objects, for which basic linear algebra operations such as addition and multiplication are available. The BEM++ library will take care of the correct mapping properties and uses mass-matrix transformations where necessary. Combined field boundary integral formulations can thus conveniently be created with the following high-level instructions.

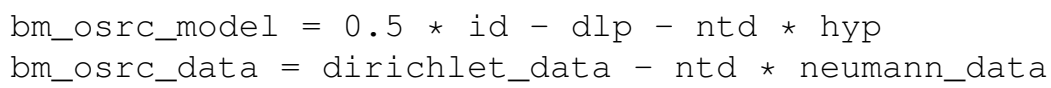

Here, we have shown the creation of the OSRC-preconditioned Burton-Miller formulation (25). Other formulations can be implemented similarly.

So far, we have defined the boundary integral formulation with abstract objects. The actual discretization of the operators is not being performed until necessary or explicitly called. Instead of calling the weak formulation, we opt to compute the strong formulation which is the weak formulation with additional mass matrix preconditioning. By default, the matrix assembly is performed with $\mathscr{H}$-matrix compression enabled. The right-hand-side vector is given by the coefficients of the excitation data.

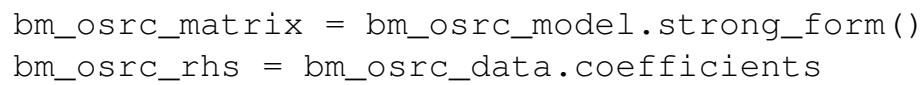

The obtained matrix and right-hand-side vector can be interpreted by the SciPy library. This allows for solving the discrete system with its GMRES implementation.

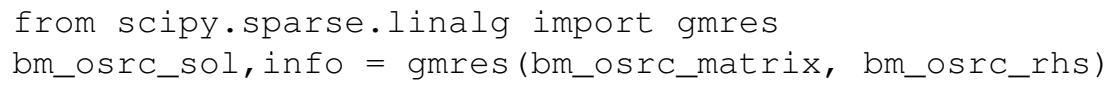


The surface potential can readily be visualized with e.g. Gmsh but BEM++ also provides functionality to compute the scattered field outside the boundary. For this, an array of locations points have to be created on which the exterior field will be computed.

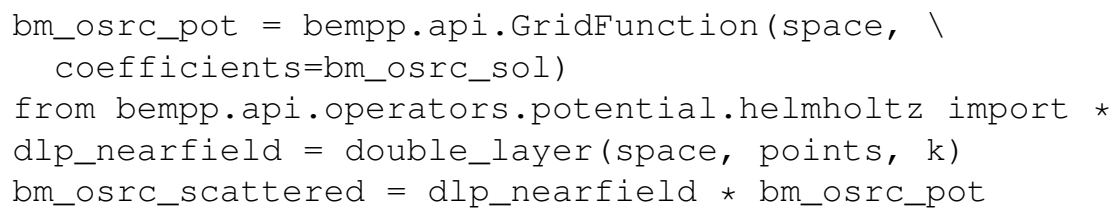

The resulting field can then be exported for further processing or directly plotted using a Python plotting library.

\subsection{Numerical results}

In this section we present some numerical results on canonical test shapes which demonstrate the performance of the formulations discussed in the previous sections. An application problem with realistic data from medical engineering will be presented in Section 6.

\subsubsection{Stability in the presence of resonances}

A prime advantage of the combined field integral equations over simpler formulations is stability at resonance frequencies. For example, the double-layer formulation (17) has a nontrivial nullspace at resonance frequencies, which are explicitly known for special geometries such as a cube. To this end, let us consider a unit-sized cube near the two resonances of $k=\pi \sqrt{1+1+3^{2}}=10.42$ and $k=\pi \sqrt{1+2^{2}+3^{2}}=11.75$. The mesh is created with an oversampling of ten elements per wavelength.

$$
\text { grid }=\text { bempp.api.shapes. cube }(h=2 * n p \cdot p i /(10 * k))
$$

The incident wave field is given by a plane wave field traveling in the positive $x$ direction and P1-elements are used for discretization. As a linear solver, the GMRES method available from the SciPy library has been used with a tolerance of 1.0E-5.

As can be seen in Fig. 2, the number of iterations used by the GMRES solver clearly depends on the choice of boundary integral formulation. The number of iterations for the Burton-Miller formulation and its preconditioned variant are constant for this small frequency range. The peaks at the resonance frequencies indicate the breakdown of the double-layer formulation. While at these low frequencies the convergence is still reasonable, this becomes problematic for high frequencies where the modal density increases. 


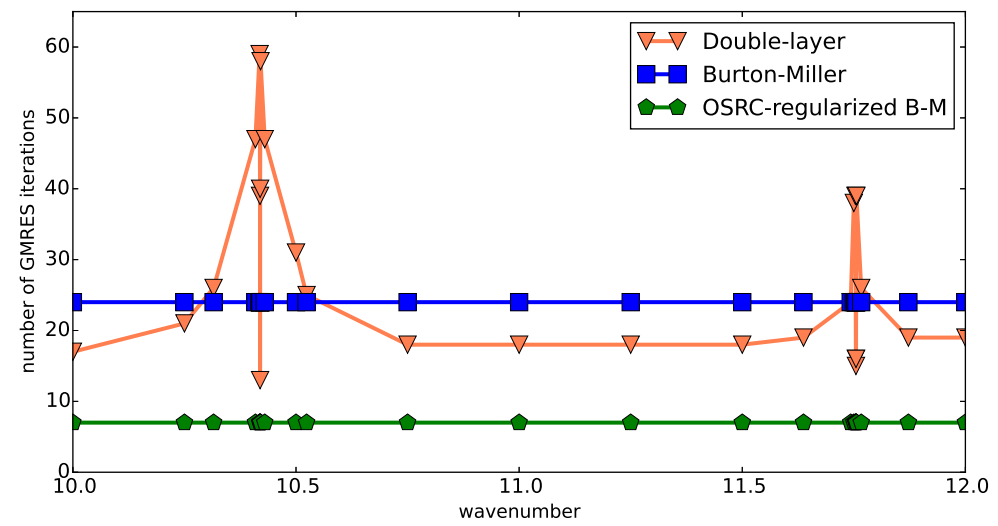

Fig. 2 The GMRES convergence for different model formulations near two resonance frequencies.

\subsubsection{Performance with frequency at an re-entrant cube}

Although the combined field formulations are stable with respect to resonances, their convergence will deteriorate when increasing the frequency. The use of regularization is expected to improve the convergence, as explained in Section 2.3. Here, we will test this on a re-entrant cube of unit dimension, meshed with an oversampling of ten elements per wavelength.

grid=bempp . api.shapes.reentrant_cube $(h=2 \star n p \cdot p i /(10 * k))$

The solution of the Burton-Miller formulation for $k=37$ has been depicted in Fig. 3 . For this wavenumber, the size of the object measures ten wavelengths across and 28068 degrees of freedom are present.

The performance with respect to frequency of four different formulations will be assessed with this test case: the Burton-Miller formulation (18), its OSRCpreconditioned variant (25), the Brakhage-Werner formulation (21), and its complexwavenumber single-layer regularized variant (27). For the standard Brakhage-Werner formulation we choose $\mathscr{R}=1 / k$ as a resemblance to the Burton-Miller formulation. As linear solver, the GMRES algorithm without restart is being used. Both the number of iterations and the wall-clock time of the linear solver are depicted in Fig. 4.

The experiment clearly shows that the use of regularization does have a big impact on the performance of the linear solver. The OSRC preconditioner and complex single-layer regularization both reduce the number of iterations considerably compared with the classical Burton-Miller and Brakhage-Werner formulations. The reduction of number of iterations with the preconditioning strategies was not achieved at the price of much computational overhead. More precisely, compared to the classical formulations, the preconditioning results in an average overhead of $1.6 \%$ and $1.8 \%$ per iteration for OSRC and complex single-layer regularization, respectively. However, both require additional initial setup time. For the OSRC this is the compu- 


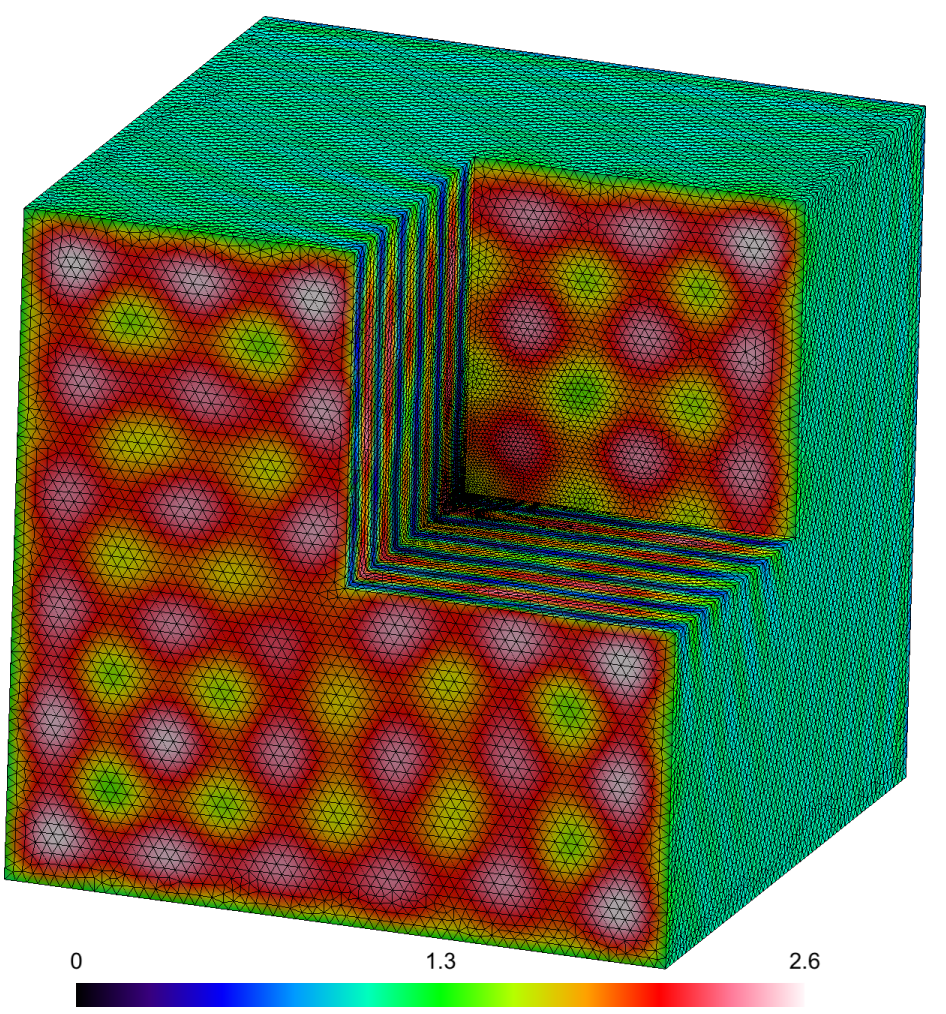

Fig. 3 The magnitude of the surface potential on the re-entrant cube for wavenumber $k=37$.

tation of sparse LU decompositions of the surface Helmholtz problems and for the complex-single layer regularization it is the $\mathscr{H}$-matrix assembly of the compressed single-layer operator. For the presented examples, both are small compared to the assembly times of the other operators involved in the Burton-Miller and BrakhageWerner formulations.

\section{HIFU treatment}

This section describes the application of the fast BEM techniques to a challenging problem of importance in medical engineering. To reduce the health risks of open surgery, clinicians are increasingly inclined to use modern non-invasive techniques, such as High-Intensity Focused Ultrasound (HIFU) treatment. Computational methods have the potential to improve the patient-specific treatment planning. Here, we will consider the case of transcostal HIFU, where the presence of the ribs has a significant influence on the sound propagation. Since the computational model is based 

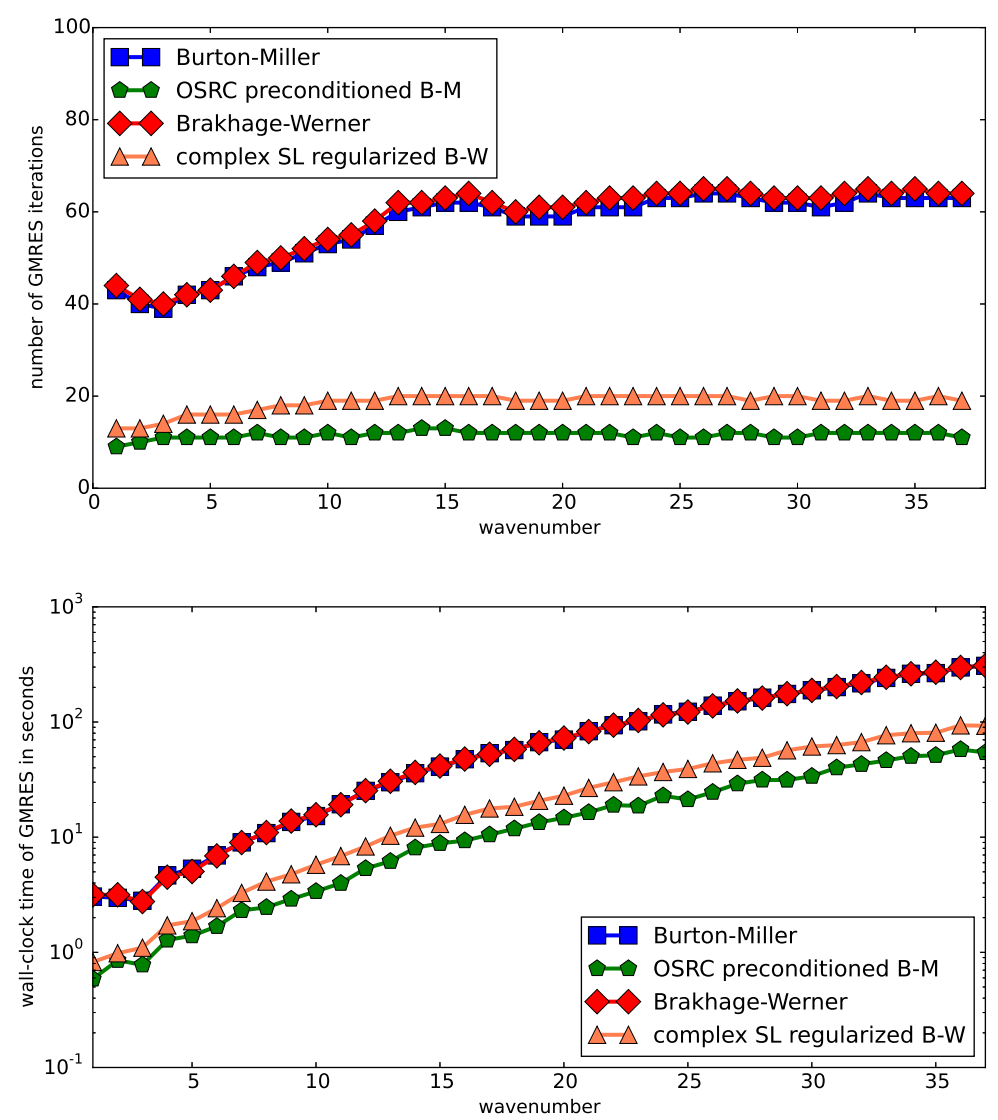

Fig. 4 The GMRES convergence for different model formulations on a re-entrant cube.

on an exterior scattering problem, the BEM is perfectly suited as numerical solution technique.

\subsection{Application to a realistic high-frequency problem in HIFU treatment}

Surgery is the most effective local therapy for treating solid malignancies [18]. However, surgery to remove tumors in specific organs, such as the liver, still presents considerable challenges [14], with prognoses for the patients remaining poor [47]. The significant negative side effects associated with surgical interventions have led to an ongoing quest for safer, more efficient and better tolerated alternatives. In recent years, there has been a notable shift away from open surgery towards less invasive 
procedures, such as laparoscopic and robotic surgery, and also energy-based methods for in situ tumor destruction. The latter include embolization, radiofrequency, microwave and laser ablation, cryoablation and HIFU [18]. HIFU is a medical procedure which uses high-amplitude ultrasound to heat and ablate a localized region of tissue. Typically, the ultrasound is generated by a focused transducer located outside the human body. As the ultrasound propagates through tissue and at high acoustic intensities, absorption of the energy can induce local tissue necrosis targeted within a well-defined volume without damaging the overlying tissue [45]. Currently, HIFU is the only non-ionizing intervention capable of completely non-invasive ablation. The clinical acceptance of HIFU has grown in recent years, leading to its FDA approval for treating uterine fibroids, prostate cancer and for the palliative treatment of bone metastases.

Whilst the feasibility of HIFU for the treatment of cancer of the liver has been demonstrated [19], there remain a number of significant challenges which currently hinder its more widespread clinical application. The liver is located in the upperright portion of the abdominal cavity under the diaphragm and to the right of the stomach. When administering a HIFU treatment in view of destroying tumors of the liver, the ultrasonic transducer is positioned outside the body and typically coupled to the abdomen via a region of water. Rib bone, which both absorbs and reflects ultrasound strongly, may therefore narrow the acoustic window between the transducer and the tumor. Hence, a common side effect of focusing ultrasound in regions located behind the rib cage is the overheating of bone and surrounding tissue, which can lead to skin burns at the ribs [39]. Furthermore, the presence of ribs can lead to aberrations at the focal region due to effects of diffraction [25].

One of the minimal technical specifications of a HIFU system for the treatment of liver tumors should be to transmit energy either in between, below, or through the ribs without damaging the ribs or causing a skin burn [46]. A means of addressing this requirement is via a patient-specific treatment planning protocol based on numerical simulations carried out using the patient's anatomical data. Such a protocol could provide a standardized framework by which HIFU may be optimized to treat tumors of the liver without adverse effects. The role of numerical models also extends to pre-clinical experiments on soft tissue and bone mimicking phantoms. As there remain substantial metrological challenges when carrying out such physical experiments, validated numerical models play a key role in planning this work and interpreting its outcome.

\subsection{Methodology}

As the ultrasonic waves propagate from the surface of the transducer to the focal region, they will encounter water and soft tissue, including skin and fat, and rib bone, before finally reaching the liver. Different soft tissue types tend to bear acoustic properties similar to those of water. The speed of propagation of longitudinal waves in these media is generally comparable, and is approximately $1500 \mathrm{~m} \cdot \mathrm{s}^{-1}$ [21]. The 
same is true of the density [21], which is around $1000 \mathrm{~kg} \cdot \mathrm{m}^{-3}$. Ribs however act as strong scatterers, owing to their higher acoustic impedance relative to that of soft tissue. A first step towards treating the problem of scattering of a HIFU field by the rib cage is therefore to consider the ribs as being immersed in an infinite homogeneous medium with acoustic properties representative of those of soft tissue. The modeling of the scattering of the field of a HIFU array by human ribs can then be considered as an exterior scattering problem. This can be efficiently treated using the BEM [26]. The optimal transducer excitation frequency for HIFU of the liver has been established to be around $1 \mathrm{MHz}-1.5 \mathrm{MHz}$. At frequencies below $1 \mathrm{MHz}$, the cavitation threshold in tissue decreases, thus creating the risk of unwanted cavitation at pre-focal regions. At frequencies above $1.5 \mathrm{MHz}$, since attenuation in soft tissue is roughly proportional to frequency, the resulting focal intensities may be too low to achieve tissue necrosis, particularly in the case of deep-seated tumors. For transcostal HIFU, this implies that the wavelengths in soft tissue will be around $1.0 \mathrm{~mm}-1.5 \mathrm{~mm}$. The computational domain being approximately $20 \mathrm{~cm} \times 20 \mathrm{~cm}$ $\times 20 \mathrm{~cm}$ reinforces the notion that it is advantageous to employ a computational method which does not rely on a volumetric mesh, which strengthens the case for using the BEM.

The advent of multi-element array transducers driven by multi-channel electronics offers significant advantages over concave single-element piezoelectric devices. Multi-element transducers have the ability to compensate for tissue and bone heterogeneities and to steer the beam electronically by adjusting the time delays in each channel to produce constructive interference at the required location, thus minimizing the requirement for mechanical repositioning of the transducer during treatment. A pseudo-random arrangement of the circular planar elements on the surface of the transducer is often opted for. This has been shown to minimize the formation of side lobes when design constraints place a limit on the amount of elements that can be used and on the spacing between these elements [24]. Fig. 5 depicts a mesh of four ribs, together with a spherical section transducer array, with 256 pseudo-randomly distributed elements. The array is positioned so that its geometric focus is located at an intercostal space, approximately $3 \mathrm{~cm}$ deep into the rib cage.

In order to address the scattering problem, a suitable description of the incident acoustic field and its normal derivative on the surface of the ribs must be arrived at. In the case of multi-element transducers, the incident acoustic pressure field is commonly modeled as a superposition of plane circular piston sources [24]. The spatial component of the acoustic field of such a circular source may be represented by the Rayleigh integral, which can be solved using numerical quadrature techniques [48].

\subsection{Computational results}

In Section 6.2, it was proposed that, in first instance, a physical model for HIFU treatment planning of the liver could be formulated as an exterior scattering problem. The BEM is ideally suited to tackle such problems. The strict requirement of 


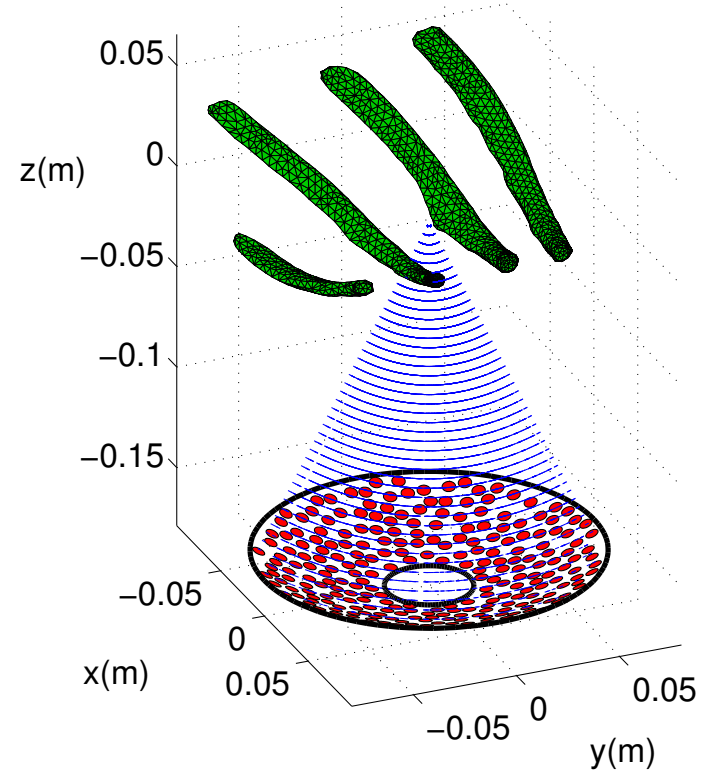

Fig. 5 Position of ribs relative to a HIFU array for an intercostal treatment, approximately $3 \mathrm{~cm}$ deep into the rib cage.

frequencies in the $\mathrm{MHz}$ range necessitates the use of fast solution techniques, such as operator preconditioning and matrix compression. Here, we will use the OSRCpreconditioned Burton-Miller formulation with $\mathscr{H}$-matrix compression since this has experimentally proved to be the most effective technique.

The scattering object is given by a human rib cage model [25], consisting of the four ribs closest to the liver. The ribs are rigid and immersed in an infinite domain where the speed of sound is $1500 \mathrm{~m} \cdot \mathrm{s}^{-1}$, as is typical for water and soft tissue. The ultrasound excitation is generated by a multi-element transducer array of 256 piston sources. The field generated by each element is modeled with a numerical quadrature rule, resulting in a total of 38144 point sources. The frequency of the ultrasound field is $1 \mathrm{MHz}$, which corresponds to a wave length of $1.5 \mathrm{~mm}$. The diameter of the ribcage model is $20.3 \mathrm{~cm}$, which makes it 135 times larger than the wave length.

The surface mesh at the ribs consists of triangles with a maximum width of $0.18 \mathrm{~mm}$, thus representing each wavelength with at least 8 elements. The boundary element space of continuous piecewise linear elements contains 479124 degrees of freedom. The experiment has been performed on a high-specification workstation of eight quad-cores with a clock rate of $2.8 \mathrm{GHz}$ each. The shared memory is $264 \mathrm{~GB}$.

Standard values for the parameters in the OSRC-preconditioner have been used, namely a size of 4 and a branch cut of $\pi / 3$ for the Padé approximation. The GMRES solver of SciPy has been used with a default termination criterion of $10^{-5}$ and finished the solution in 19 iterations and 6:59 minutes only. 
The assembly of the dense matrices has been performed with $\mathscr{H}$-matrix compression with an $\mathcal{E}$-value of $10^{-5}$, a maximum rank of 1000 and a maximum block size of 100000 . The assembly of the boundary operators took 5 hours and 16 minutes. Where the storage of dense matrices would have needed in excess of $7 \mathrm{~TB}$ memory, the compressed matrices required 194 GB only. The compression rates are $2.08 \%$ and $3.31 \%$ for the single-layer and hypersingular boundary operator, respectively.

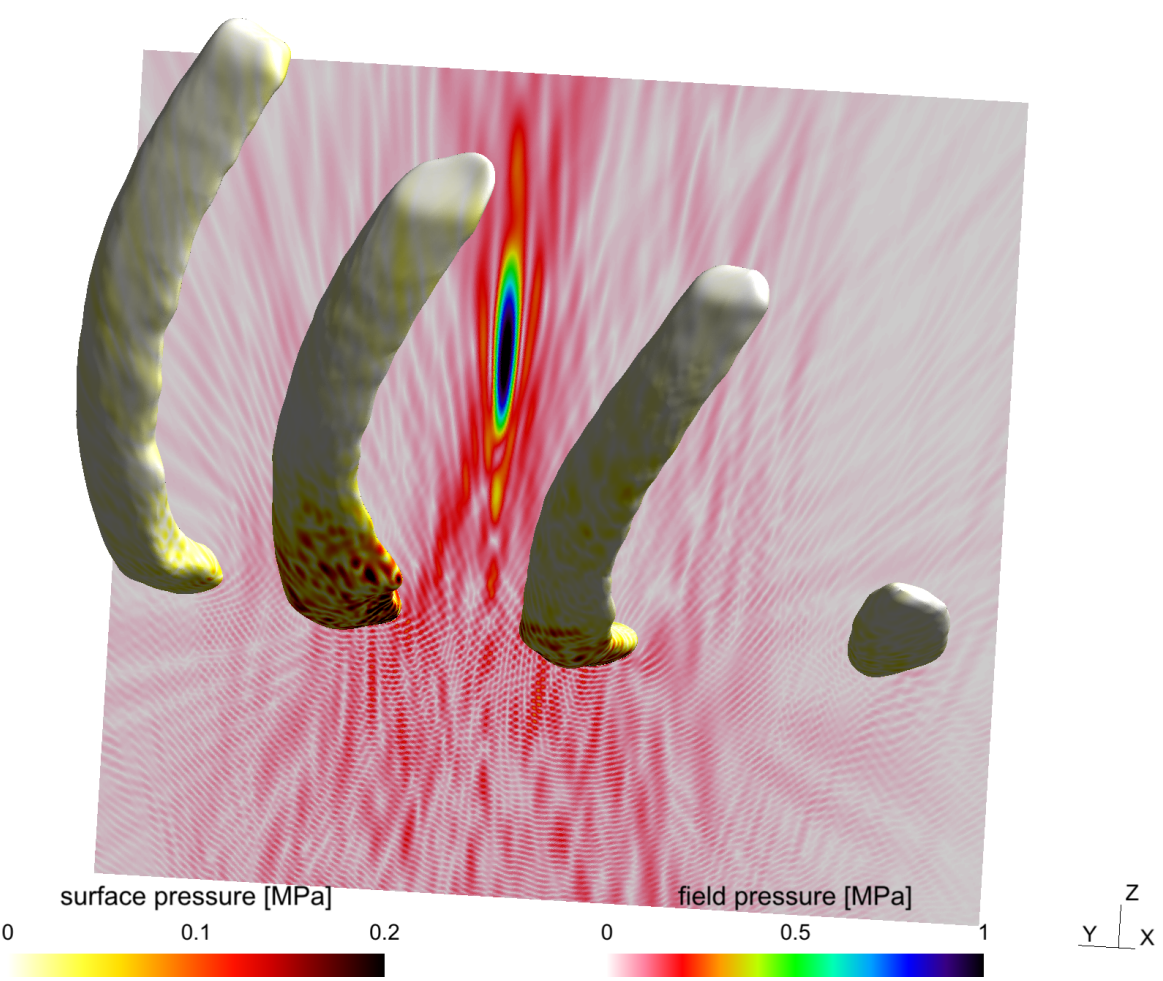

Fig. 6 The computational results of the HIFU model. At the surface the magnitude of the surface potential $\varphi=\left.u^{\mathrm{tot}}\right|_{\Gamma}$ and on the exterior plane with $x=0$ the magnitude of the total wave field $u^{\text {inc }}+\mathscr{K} \varphi=u^{\text {tot }}$ have been visualized.

The total field exterior to the rib cage was computed on a vertical plane and is visualized in Fig. 6. The reflected waves are clearly visible, along with a shadow region behind the ribs. The influence of the scattering on the focal region is not significant in this configuration: the energy is still bundled in the desired region. The realistic wave field for this challenging object confirms the capability of our modern BEM implementation to simulate acoustic scattering at high frequencies. 


\section{Discussion}

In this chapter we have demonstrated efficient BEM formulations for exterior acoustic problems, their fast implementation using the open-source BEM++ library, and performance results when applied to a realistic high-frequency problem. Modern preconditioning strategies for the Burton-Miller formulation based on OSRC or complex wavenumber single-layer boundary operators are highly effective and lead to a small number of GMRES iterations for each right-hand side. Even though the applicability of the BEM to large-scale simulations has been confirmed in this chapter, there is still a need for faster computations. A goal is to incorporate the BEM in an optimization routine for the configuration of HIFU transducer arrays. This necessitates the solution of the BEM formulation for multiple right-hand-side vectors. When such an implementation could be achieved effectively, this would bring the BEM a step closer to actual application in a clinical environment.

Significant speed improvements are still possible with respect to the discretization of the boundary operators. While the $\mathscr{H}$-matrix based discretization described in this chapter performs well for many Helmholtz problems, a direct improvement is possible by moving towards $\mathscr{H}^{2}$-matrix techniques. They allow for a considerable memory reduction [8], but similar to $\mathscr{H}$-matrices, they are not asymptotically optimal at high frequencies.

For problems with only few right-hand sides, high-frequency FMM methods [16, 30] are very efficient. Yet, they are less suited for problems with many right-hand sides due to their often slower matrix-vector product. Wideband hierarchical matrix techniques such as the one presented in [6] combine fast algebraic matrix-vector products with asymptotic optimal complexity as $k \rightarrow \infty$.

A potential improvement to the limitations at high-frequencies may be the development of fast approximate direct solvers. While there has been considerable progress for low-frequency problems (see e.g. [10]), the development of fast approximate direct solvers that scale well as $k \rightarrow \infty$ remains elusive. The most promising approach may be based on butterfly compression techniques. A butterfly recompression scheme for an approximate $\mathscr{H}$-matrix LU decomposition is described in [31]. The results in this paper are impressive but still require an initial compression using standard $\mathscr{H}$-matrices.

While there is a wealth of software available for finite element discretizations there are still few open-source packages for boundary element problems. The $\mathrm{BEM}++$ library is continuously being developed and aims to integrate modern technologies as they become relevant for practical applications. We have given a demonstration of BEM++ in this chapter. Many more example applications including Maxwell problems are described at the website www.bempp.org. 


\section{References}

1. S. Amini and P. J. Harris. A comparison between various boundary integral formulations of the exterior acoustic problem. Computer methods in applied mechanics and engineering, 84(1):59-75, 1990.

2. S. Amini and Stephen Martin Kirkup. Solution of Helmholtz equation in the exterior domain by elementary boundary integral methods. Journal of Computational Physics, 118(2):208221, 1995.

3. S. Amini and N. D. Maines. Preconditioned Krylov subspace methods for boundary element solution of the Helmholtz equation. International Journal for Numerical Methods in Engineering, 41(5):875-898, 1998.

4. Xavier Antoine and Marion Darbas. Alternative integral equations for the iterative solution of acoustic scattering problems. The Quarterly Journal of Mechanics and Applied Mathematics, 58(1):107-128, 2005

5. Xavier Antoine and Marion Darbas. Generalized combined field integral equations for the iterative solution of the three-dimensional Helmholtz equation. ESAIM: Mathematical Modelling and Numerical Analysis, 41(01):147-167, 2007.

6. M. Bebendorf, C. Kuske, and R. Venn. Wideband nested cross approximation for Helmholtz problems. Numerische Mathematik, 130(1):1-34, jul 2014.

7. Mario Bebendorf. Hierarchical matrices. Springer, 2008.

8. Steffen Börm. Efficient numerical methods for non-local operators, volume 14 of EMS Tracts in Mathematics. European Mathematical Society (EMS), Zürich, 2010. $\mathscr{H}^{2}$-matrix compression, algorithms and analysis.

9. Helmut Brakhage and Peter Werner. Über das Dirichletsche Aussenraumproblem für die Helmholtzsche Schwingungsgleichung. Archiv der Mathematik, 16(1):325-329, 1965.

10. James Bremer, Adrianna Gillman, and Per-Gunnar Martinsson. A high-order accurate accelerated direct solver for acoustic scattering from surfaces. BIT Numerical Mathematics, 55(2):367-397, jul 2014.

11. Oscar Bruno, Tim Elling, and Catalin Turc. Regularized integral equations and fast high-order solvers for sound-hard acoustic scattering problems. International Journal for Numerical Methods in Engineering, 91(10):1045-1072, 2012.

12. A. Buffa and R. Hiptmair. Regularized combined field integral equations. Numerische Mathematik, 100(1):1-19, 2005.

13. A. J. Burton and G. F. Miller. The application of integral equation methods to the numerical solution of some exterior boundary-value problems. Proceedings of the Royal Society of London A: Mathematical, Physical and Engineering Sciences, 323(1553):201-210, 1971.

14. Charles H. Cha, M. Wasif Saif, Brett H. Yamane, and Sharon M. Weber. Hepatocellular carcinoma: Current management. Current Problems in Surgery, 47(1):10-67, 2010.

15. Simon N. Chandler-Wilde, Ivan G. Graham, Stephen Langdon, and Euan A. Spence. Numerical-asymptotic boundary integral methods in high-frequency acoustic scattering. Acta Numerica, 21:89-305, apr 2012.

16. H Cheng, W Y Crutchfield, Z Gimbutas, L F Greengard, J F Ethridge, J Huang, V Rokhlin, $\mathrm{N}$ Yarvin, and $\mathrm{J}$ Zhao. A wideband fast multipole method for the Helmholtz equation in three dimensions. Journal of Computational Physics, 216(1):300-325, 2006.

17. Weng Cho Chew, Eric Michielssen, J. M. Song, and Jian-Ming Jin. Fast and efficient algorithms in computational electromagnetics. Artech House, Inc., 2001.

18. David Cranston. A review of high intensity focused ultrasound in relation to the treatment of renal tumours and other malignancies. Ultrasonics Sonochemistry, 27:654-658, 2015.

19. Lawrence Crum, Michael Bailey, Joo Ha Hwang, Vera Khokhlova, and Oleg Sapozhnikov. Therapeutic ultrasound: Recent trends and future perspectives. Physics Procedia, 3(1):25-34, 2010. International Congress on Ultrasonics, Santiago de Chile, January 2009.

20. Marion Darbas, Eric Darrigrand, and Yvon Lafranche. Combining analytic preconditioner and fast multipole method for the 3-D Helmholtz equation. Journal of Computational Physics, 236:289-316, 2013. 
21. Francis A. Duck. Physical properties of tissues - A comprehensive reference book. Academic, 1990.

22. B. Engquist and H. Zhao. Approximate Separability of Green's Function for High Frequency Helmholtz Equations. Technical report.

23. Björn Engquist and Lexing Ying. Fast directional multilevel algorithms for oscillatory kernels. SIAM J. Sci. Comput., 29(4):1710-1737 (electronic), 2007.

24. Leonid R. Gavrilov and Jeffrey W. Hand. High-Power Ultrasound Phased Arrays for Medical Applications. Nova, 2014.

25. P. Gélat, G. ter Haar, and N. Saffari. A comparison of methods for focusing the field of a HIFU array transducer through human ribs. Physics in Medicine and Biology, 59(12):3139-3171, 2014.

26. Pierre Gélat, Gail ter Haar, and Nader Saffari. Modelling of the acoustic field of a multielement HIFU array scattered by human ribs. Physics in Medicine and Biology, 56(17):55535581, 2011.

27. Christophe Geuzaine and Jean-François Remacle. Gmsh: A 3-D finite element mesh generator with built-in pre-and post-processing facilities. International Journal for Numerical Methods in Engineering, 79(11):1309-1331, 2009.

28. Dan Givoli. Computational absorbing boundaries. In Computational Acoustics of Noise Propagation in Fluids-Finite and Boundary Element Methods, pages 145-166. Springer, 2008.

29. I. G. Graham, M. Löhndorf, J. M. Melenk, and E. A. Spence. When is the error in the h-BEM for solving the Helmholtz equation bounded independently of $\mathrm{k}$ ? BIT Numerical Mathematics, 55(1):171-214, sep 2014.

30. Nail A Gumerov and Ramani Duraiswami. A broadband fast multipole accelerated boundary element method for the three dimensional Helmholtz equation. The Journal of the Acoustical Society of America, 125(1):191-205, 2009.

31. Han Guo, Jun $\mathrm{Hu}$, and Eric Michielssen. On MLMDA/Butterfly Compressibility of Inverse Integral Operators. IEEE Antennas and Wireless Propagation Letters, 12:31-34, 2013.

32. Wolfgang Hackbusch. Hierarchical matrices: Algorithms and Analysis. Springer, 2015.

33. R. Hiptmair. Operator Preconditioning. Computers \& Mathematics with Applications, 52(5):699-706, sep 2006.

34. Ralf Hiptmair. Operator preconditioning. Computers and mathematics with Applications, 52(5):699-706, 2006.

35. Maryna Kachanovska. Hierarchical matrices and the high-frequency fast multipole method for the Helmholtz equation with decay. Technical Report 13, MPI Leipzig, 2014.

36. Robert C. Kirby. From functional analysis to iterative methods. SIAM review, 52(2):269-293, 2010.

37. Rainer Kress. Minimizing the condition number of boundary integral operators in acoustic and electromagnetic scattering. The Quarterly Journal of Mechanics and Applied Mathematics, 38(2):323-341, 1985.

38. Marc Lenoir and Nicolas Salles. Evaluation of 3-D Singular and Nearly Singular Integrals in Galerkin BEM for Thin Layers. SIAM Journal on Scientific Computing, 34(6):A3057-A3078, jan 2012.

39. Jun-Lun Li, Xiao-Zhou Liu, Dong Zhang, and Xiu-Fen Gong. Influence of ribs on the nonlinear sound field of therapeutic ultrasound. Ultrasound in Medicine and Biology, 33(9):14131420, 2007.

40. Jean-Claude Nédélec. Acoustic and electromagnetic equations: integral representations for harmonic problems, volume 144. Springer Science \& Business Media, 2001.

41. Stefan A. Sauter and Christoph Schwab. Boundary element methods, volume 39 of Springer Series in Computational Mathematics. Springer-Verlag, Berlin, 2011.

42. Wojciech Śmigaj, Timo Betcke, Simon Arridge, Joel Phillips, and Martin Schweiger. Solving boundary integral problems with BEM++. ACM Transactions on Mathematical Software (TOMS), 41(2):6, 2015.

43. O. Steinbach and W.L. Wendland. The construction of some efficient preconditioners in the boundary element method. Advances in Computational Mathematics, 9(1-2):191-216, 1998. 
44. Olaf Steinbach. Numerical approximation methods for elliptic boundary value problems: finite and boundary elements. Springer Science \& Business Media, 2007.

45. G. ter Haar, D. Sinnett, and I. Rivens. High intensity focused ultrasound-a surgical technique for the treatment of discrete liver tumours. Physics in Medicine and Biology, 34(11):17431750, 1989.

46. J.-F. Aubry et al. Transcostal high-intensity-focused ultrasound: ex vivo adaptive focusing feasibility study. Journal of Therapeutic Ultrasound, 1:1-13, 2013.

47. James S. Tomlinson et al. Actual 10-year survival after resection of colorectal liver metastases defines cure. Journal of Clinical Oncology, 25(29):4575-4580, 2007.

48. Elwin van 't Wout, Pierre Gélat, Timo Betcke, and Simon Arridge. A fast boundary element method for the scattering analysis of high-intensity focused ultrasound. The Journal of the Acoustical Society of America, 138(5):2726-2737, 2015.

49. Jianlin Xia, Shivkumar Chandrasekaran, Ming Gu, and Xiaoye S. Li. Fast algorithms for hierarchically semiseparable matrices. Numerical Linear Algebra with Applications, 17(6):953976, dec 2010. 